\title{
Effect of severe left ventricular systolic dysfunction on hospital outcome after transcatheter aortic valve implantation or surgical aortic valve replacement: Results from a propensity-matched population of the Italian OBSERVANT multicenter study
}

Francesco Onorati, MD, PhD, ${ }^{\mathrm{a}}$ Paola D'Errigo, MSc, ${ }^{\mathrm{b}}$ Claudio Grossi, MD,${ }^{\mathrm{c}}$ Marco Barbanti, MD,${ }^{\mathrm{d}}$ Marco Ranucci, MD, ${ }^{\mathrm{e}}$ Daniel Remo Covello, MD, ${ }^{\mathrm{f}}$ Stefano Rosato, MSc, ${ }^{\mathrm{b}}$ Alice Maraschini, MSc, ${ }^{\mathrm{b}}$ Gennaro Santoro, MD, ${ }^{\mathrm{g}}$ Corrado Tamburino, MD, ${ }^{\mathrm{d}}$ Fulvia Seccareccia, MSc, ${ }^{\mathrm{b}}$ Francesco Santini, MD, ${ }^{\mathrm{h}}$ and Lorenzo Menicanti, MD, ${ }^{e}$ on behalf of the OBSERVANT Research Group

Objective: Despite demonstration of the superior outcomes of transcatheter aortic valve implantation (TAVI) versus optimal medical therapy for severe left ventricular systolic dysfunction, studies comparing TAVI and surgical aortic valve replacement (AVR) in this high-risk group have been lacking.

Methods: We performed propensity matching for age, gender, baseline comorbidities, previous interventions, priority at hospital admission, frailty score, New York Heart Association class, EuroSCORE, and associated cardiac diseases. Next, the 30-day mortality and procedure-related morbidity of 162 patients (81 TAVI vs 81 AVR) with severe left ventricular systolic dysfunction (ejection fraction $\leq 35 \%$ ) were analyzed at the Italian National Institute of Health.

Results: The 30-day mortality was comparable $(P=.37)$ between the 2 groups. The incidence of periprocedural acute myocardial infarction $(P=.55)$, low output state $(P=.27)$, stroke $(P=.36)$, and renal dysfunction (peak creatinine level, $P=.57$ ) was also similar between the 2 groups. TAVI resulted in significantly greater postprocedural permanent pacemaker implantation $(P=.01)$ and AVR in more periprocedural transfusions $(P<.01)$ despite a similar transfusion rate per patient ( $2.8 \pm 3.7$ for TAVI vs $4.4 \pm 3.8$ for AVR; $P=.08)$. The postprocedural intensive care unit stay (median, 2 days after TAVI vs 3 days after AVR; $P=.34$ ), intermediate care unit stay (median, 0 days after both TAVI and AVR; $P=.94$ ), and hospitalization (median, 11 days after TAVI vs 14 days after AVR; $P=.51$ ) were comparable.

Conclusions: In patients with severe left ventricular systolic dysfunction, both TAVI and AVR are valid treatment options, with comparable hospital mortality and periprocedural morbidity. Comparisons of the mid- to long-term outcomes are mandatory. (J Thorac Cardiovasc Surg 2014;147:568-75)

The coexistence of severe aortic stenosis (SAS) and severe left ventricular systolic dysfunction (SLVSD) significantly affects the prognosis and increases the perioperative risk

From the Division of Cardiac Surgery, ${ }^{\mathrm{a}}$ University of Verona Medical School, Verona, Italy; National Centre for Epidemiology, Surveillance and Health Promotion, ${ }^{\mathrm{b}}$ Istituto Superiore di Sanità, Rome, Italy; Department of Cardiovascular Surgery, S. Croce e Carle Hospital, Cuneo, Italy; Division of Cardiology, ${ }^{\mathrm{d}}$ Ferrarotto Hospital, University of Catania, Italy, and Excellence Through Newest Advances Foundation, Catania, Italy; Department of Cardiothoracic and Vascular Anesthesia-Intensive Care Unit and Department of Cardiac Surgery, ${ }^{\mathrm{e}}$ IRCCS Policlinico San Donato, San Donato Milanese, Milan, Italy; Department of Anesthesia and Intensive Care, ${ }^{f}$ IRCCS San Raffaele, Milan, Italy; Division of Cardiology, ${ }^{g}$ Careggi Hospital, Florence, Italy; and Division of Cardiac Surgery, ${ }^{\text {h }}$ University Hospital San Martino, Genoa, Italy.

Disclosures: Authors have nothing to disclose with regard to commercial support.

Read at the 39th Annual Meeting of The Western Thoracic Surgical Association, Coeur d'Alene, Idaho, June 26-29, 2013.

Received for publication July 2, 2013; revisions received Sept 17, 2013; accepted for publication Oct 6, 2013; available ahead of print Nov 21, 2013.

Address for reprints: Francesco Onorati, MD, PhD, Division of Cardiac Surgery, University of Verona Medical School, Verona, Italy (E-mail: frankono@libero.it).

$0022-5223 / \$ 36.00$

Copyright (c) 2014 by The American Association for Thoracic Surgery

http://dx.doi.org/10.1016/j.jtcvs.2013.10.006 of surgical aortic valve replacement (AVR) according to both the EuroSCORE and the Society of Thoracic Surgeons score. ${ }^{1}$ Although AVR still represents the reference standard to cure SAS in the presence of SLVSD because of the demonstrated survival benefit in patients without or with uncertain inotropic reserve, ${ }^{2}$ the combination of SLVSD with advanced age and significant comorbidities could occasionally result in a predicted operative risk great enough to potentially outweigh the survival benefits of AVR. ${ }^{3}$ Accordingly, since the demonstration of the safety and efficacy of transcatheter aortic valve implantation (TAVI) to cure SAS in patients at high risk of AVR, the application of TAVI has widened to include the subset of patients with SLVSD. ${ }^{4-7}$ However, contradictory results have been reported in TAVI studies, with some studies showing comparable early and long-term outcomes, regardless of the preoperative systolic left ventricular function, ${ }^{4,5}$ some reporting comparable survival but a greater incidence of major adverse cardiovascular events in patients with depressed ventricular function, ${ }^{6}$ and some showing significantly better left ventricular ejection 


\section{Abbreviations and Acronyms \\ AMI = acute myocardial infarction \\ AVR = aortic valve replacement \\ FRANCE-2 = French Transcatheter Aortic Valve Intervention

$\begin{array}{ll}\text { ICU } & =\text { intensive care unit } \\ \text { ImCU } & =\text { intermediate care unit } \\ \text { LCOS } & \text { low cardiac output syndrome } \\ \text { LVEF } & \text { left ventricular ejection fraction } \\ \text { PARTNER = } & \text { Placement of AoRTic TraNscathetER } \\ \text { PPM } & \text { permanent pacemaker } \\ \text { SAS } & =\text { severe aortic stenosis } \\ \text { SLVSD }= & \text { severe left ventricular systolic } \\ & \text { dysfunction } \\ \text { TAVI } & \text { transcatheter aortic valve } \\ & \text { implantation } \\ \text { VARC } & \text { Valve Academic Research } \\ & \text { Consortium }\end{array}$

fraction (LVEF) recovery despite lower 30-day and 1-year survival of patients with SLVSD. ${ }^{7}$ Furthermore, most of these studies were retrospective analyses of singlecenter experiences or that of a few centers. ${ }^{4-6}$ Therefore, despite the widespread use of TAVI for SLVSD, studies directly comparing AVR and TAVI in this high-risk cohort have been lacking, possibly because of the different baseline risk profile of the TAVI and AVR populations. Finally, the recent analysis of the Placement of AoRTic TraNscathetER (PARTNER) valve trial of patients with low-flow severe aortic stenosis reported comparable mortality between high-risk patients randomized to surgical AVR or TAVI. ${ }^{8}$ Thus, despite the recent data from randomized studies suggesting substantial equipoise between AVR and TAVI in terms of mortality in patients with depressed ventricular function, ${ }^{8}$ the results coming from registrieswhich mirror "real-world" practice_are still lacking. Therefore, it was the aim of the present study to investigate the role of SLVSD on both clinical presentation and hospital outcomes after TAVI and AVR in a propensity-matched population. The analysis was performed from a prospective series of patients enrolled in the Italian National Institute of Health Observational Multicenter (OBSERVANT) registry, a prospective registry aimed at evaluating the efficacy and effectiveness of TAVI versus AVR for the treatment of severe SAS. ${ }^{9-11}$

\section{METHODS}

\section{Study Design and Population Enrolled}

The Italian National Health Institution, in cooperation with the Italian Ministry of Health, the National Agency for Regional Health Services, Italian regions, and Italian scientific societies representing the professionals involved in the treatment of patients with SAS started the Observational study of appropriateness, efficacy, and effectiveness of AVR-TAVI procedures for the treatment of SAS (OBSERVANT) in January 2011. Details of the OBSERVANT registry have been previously reported. ${ }^{9,11}$

In brief, on the basis of established criteria, the study included all symptomatic adult patients admitted to hospitals with a diagnosis of SAS (defined as an aortic valve area $<1 \mathrm{~cm}^{2}$, maximum aortic velocity $>4$ $\mathrm{m} / \mathrm{s}$, or mean pressure gradient $>40 \mathrm{~mm} \mathrm{Hg}$ ) and requiring an aortic valve procedure. ${ }^{9,11}$ Treatment allocation was always from review by the local multidisciplinary "heart" team involving cardiologists, surgeons, and anesthesiologists and was in accordance with the established criteria (clinical evaluation, imaging findings, and risk profile) and current guidelines. ${ }^{9}$ Although the treatment allocation stemmed from the local "heart team" evaluation, a greater EuroSCORE, more advanced age, chronic obstructive pulmonary disease, peripheral arteriopathy, previous cardiac surgery, greater frailty, advanced New York Heart Association class, concurrent coronary disease, concurrent moderate or severe mitral regurgitation, lower gradients despite a similar LVEF, all preferentially indicated TAVI for the population of patients with a LVEF of $\leq 35 \%$. Thus, all these baseline characteristics were prevalent in the TAVI cohort (Table 1).

Given the observational nature of the present study, all the percutaneous and surgical procedures were enrolled, regardless of the prosthesis employed, the surgical or percutaneous access used, and the periprocedural management.

Although the enrollment of patients in the OBSERVANT registry ended in June 2012, the present analysis included the first 12 months of data collection and focused on all patients with SAS and SLVSD enrolled at that time. SLVSD was defined by the presence of a preoperative LVEF of $\leq 35 \%$ (Simpson method) on the preoperative echocardiogram. For the purposes of the present study, a porcelain aorta, difficult thoracic approach, and frailty score of 3 (not self-sufficient) were exclusion criteria, because they preferentially contraindicated AVR and therefore did not fit with the propensity-matching analysis. The local ethics committees approved the study protocol, and all patients enrolled in the database provided informed consent to the scientific treatment of their data in an anonymous form. ${ }^{9-11}$

The endpoints of OBSERVANT registry have been previously reported. ${ }^{9,11}$ In brief, the 30-day mortality was the primary endpoint of the study. ${ }^{9-11}$ From previously published data, 30-day mortality has been recognized to be strictly related to the index procedure to be considered "real" procedural mortality. This was also in accordance with the last Valve Academic Research Consortium (VARC)-2 definitions. ${ }^{12}$ The secondary endpoints were acute myocardial infarction (AMI), stroke, major vascular complications, cardiac tamponade, transfusions (percentage of transfused patients; number of transfusions/patient), need for permanent pacemaker (PPM) implantation, low cardiac output state (LCOS), peak postoperative creatinine, postprocedural mean transprosthetic gradients and residual aortic valve regurgitation, and length of hospitalization, intensive care unit (ICU) stay, and intermediate care unit (ImCU) stay, as previously reported ${ }^{9-11}$ and in accordance with the guidelines. ${ }^{12}$ The endpoints were all adjudicated by 2 independent investigators. ${ }^{10,11}$

\section{Statistical Analysis}

All the analyses were performed, stratifying by intervention type (AVR vs TAVI). To reduce the effect of selection bias and potential confounding factors, all the outcome parameters were adjusted using the propensity score method and stepwise logistic regression (probability of enter $=0.20$; probability of removal $=0.10$ ). Nonparsimonious propensity score matching was built that included age, gender, weight, preoperative creatinine, preoperative serum albumin and hemoglobin, diabetes mellitus, chronic dialysis treatment, previous AMI and unstable angina, chronic obstructive pulmonary disease and/or preoperative oxygen dependency, neurologic dysfunction, chronic liver disease, peripheral arteriopathy, previous cardiac or vascular surgery, frailty score 1 or 2 (geriatric status scale), previous percutaneous coronary interventions, previous aortic balloon 
TABLE 1. Baseline characteristics of population with severe left ventricular systolic dysfunction (LVEF $\leq \mathbf{3 5} \%$ )

\begin{tabular}{|c|c|c|c|}
\hline Variable & $\begin{array}{c}\text { AVR } \\
(n=169)\end{array}$ & $\begin{array}{c}\text { TAVI } \\
(\mathbf{n}=\mathbf{1 8 0})\end{array}$ & $P$ value \\
\hline \multicolumn{4}{|c|}{ Demographic and clinical characteristics } \\
\hline Age (y) & $73.5 \pm 9.1$ & $79.9 \pm 8.1$ & $<.01$ \\
\hline Female gender & $91(33.8)$ & $63(35.0)$ & .07 \\
\hline Weight $(\mathrm{kg})$ & $74.3 \pm 15.4$ & $70.2 \pm 18.3$ & .01 \\
\hline Diabetes & $70(26.0)$ & $59(32.8)$ & .12 \\
\hline Creatinine $(\mathrm{mg} / \mathrm{dL})$ & $1.5 \pm 1.3$ & $1.5 \pm 1.0$ & .93 \\
\hline Chronic dialysis treatment & $9(3.3)$ & $6(3.3)$ & .96 \\
\hline Albumin (mg/dL) & $3.5 \pm 0.9$ & $3.5 \pm 0.9$ & .65 \\
\hline Hemoglobin (mg/dL) & $12.4 \pm 1.6$ & $11.5 \pm 1.7$ & $<.01$ \\
\hline Previous AMI & $17(6.3)$ & $10(5.6)$ & .95 \\
\hline Unstable angina & $10(3.7)$ & $13(7.2)$ & .10 \\
\hline COPD & $22(8.2)$ & $55(30.6)$ & $<.01$ \\
\hline Oxygen dependency & $9(3.3)$ & $14(7.8)$ & .10 \\
\hline Neurologic dysfunction & $11(4.1)$ & $13(7.2)$ & .14 \\
\hline Chronic liver disease & $6(2.2)$ & $4(2.2)$ & .47 \\
\hline Peripheral arteriopathy & $30(11.1)$ & $52(28.9)$ & .00 \\
\hline Previous cardiac surgery & $47(17.5)$ & $49(27.2)$ & .01 \\
\hline Previous vascular surgery & $7(2.6)$ & $10(5.6)$ & .26 \\
\hline Frailty score & & & $<.01$ \\
\hline 1 (self-sufficient) & $247(91.8)$ & $125(69.4)$ & \\
\hline $\begin{array}{l}2 \text { (partially } \\
\text { self-sufficient) }\end{array}$ & $22(8.2)$ & $55(30.6)$ & \\
\hline Previous PCI & $37(13.7)$ & $65(36.1)$ & $<.01$ \\
\hline $\begin{array}{l}\text { Previous aortic } \\
\text { balloon plasty }\end{array}$ & $13(4.8)$ & 43 (23.9) & $<.01$ \\
\hline Critical preoperative state & $24(8.9)$ & $24(13.3)$ & .14 \\
\hline NHYA class & & & $<.01$ \\
\hline III & $66(24.5)$ & $92(51.1)$ & \\
\hline IV & $30(11.1)$ & $44(24.4)$ & \\
\hline Logistic EuroSCORE & $15.7 \pm 13.3$ & $25.8 \pm 18.3$ & $<.01$ \\
\hline \multicolumn{4}{|c|}{ Angiographic and echocardiographic findings } \\
\hline Coronary artery disease & & & $<.01$ \\
\hline 1 -vessel & $26(9.7)$ & $46(25.6)$ & \\
\hline 2 -vessel & $12(4.5)$ & $14(7.8)$ & \\
\hline 3-vessel & $32(11.9)$ & $20(11.9)$ & \\
\hline Left main & $0(0.0)$ & $1(0.6)$ & \\
\hline Mitral valve regurgitation & & & $<.01$ \\
\hline None or mild & $222(82.5)$ & $80(44.5)$ & \\
\hline Moderate or severe & $47(17.5)$ & $100(55.5)$ & \\
\hline \multicolumn{4}{|l|}{ Aortic valve pattern } \\
\hline Valve area $\left(\mathrm{cm}^{2}\right)$ & $0.8 \pm 0.3$ & $0.7 \pm 0.3$ & $<.01$ \\
\hline Peak gradient (mm Hg) & $67.5 \pm 23.8$ & $61.9 \pm 20.8$ & $<.01$ \\
\hline Mean gradient (mm Hg) & $41.3 \pm 15.8$ & $36.9 \pm 13.3$ & $<.01$ \\
\hline Annulus diameter $(\mathrm{cm})$ & $22.1 \pm 3.2$ & $22.7 \pm 2.4$ & .06 \\
\hline \multicolumn{4}{|l|}{ Procedural characteristics } \\
\hline Urgency status & & & .31 \\
\hline Urgent & $12(4.5)$ & $15(8.3)$ & \\
\hline Emergent & $5(1.9)$ & $4(2.2)$ & \\
\hline Anesthesia & & & $<.01$ \\
\hline General & $266(98.9)$ & $62(34.4)$ & \\
\hline Epidural or local & $2(0.7)$ & $117(65.0)$ & \\
\hline Associated coronary procedure & $58(21.6)$ & $7(3.9)$ & $<.01$ \\
\hline
\end{tabular}

TABLE 1. Continued

\begin{tabular}{lccc}
\hline & AVR & TAVI & \\
Variable & $(\mathbf{n}=\mathbf{1 6 9})$ & $(\mathbf{n}=\mathbf{1 8 0})$ & $\boldsymbol{P}$ value \\
\hline Type of TAVI prosthesis & & & - \\
CV & - & $120(66.7)$ & \\
ELS & - & $60(33.3)$ &
\end{tabular}

Data presented as mean \pm standard deviation or $\mathrm{n}(\%) . L V E F$, Left ventricular ejection fraction; $A V R$, aortic valve replacement; TAVI, transcatheter aortic valve implantation; $A M I$, acute myocardial infarction; $C O P D$, chronic obstructive pulmonary disease; $P C I$, percutaneous coronary intervention; NYHA, New York Health Association; $C V$, CoreValve; $E L S$, Edwards SAPIEN valve.

plasty, critical preoperative state, New York Heart Association class, logistic EuroSCORE, associated and extent of coronary artery disease, associated and degree of mitral valve disease, aortic valve area on echocardiography, and priority status at admission. Pairs of TAVI and AVR patients with the same probability score (caliper match with 3-digit approximation) were matched. Categorical variables are presented as counts and percentages and were compared with the chi-square test or Fisher's exact test, when appropriate. Continuous variables are presented as the mean \pm standard deviations and were compared using the Student $t$ test. The statistical package, STATA, version 11 (StataCorp, College Station, Tex), was used. $P<.05$ was considered significant.

\section{RESULTS}

Of the 5890 patients prospectively enrolled in the OBSERVANT registry after 12 months, $458(7.8 \%)$ demonstrated preoperative SLVSD (LVEF $\leq 35 \%$ ). Thus, only these 458 patients were considered in the present propensity-matching analysis. The propensity matching finally selected 162 propensity-matched patients (81 for each group). However, the entire population of patients with SLVSD undergoing TAVI demonstrated significant differences compared with those undergoing AVR for most of the preoperative clinical, echocardiographic, and angiographic characteristics (Table 1). In particular, when the preoperative echocardiographic and angiographic findings were considered, patients undergoing TAVI had a greater prevalence of coronary artery disease (in particular, single-vessel, 2-vessel, and left main disease, although they underwent fewer concomitant myocardial revascularization procedures), a greater prevalence of moderate-to-severe mitral regurgitation, and a lower aortic valve area and lower peak and mean gradients, despite a trend toward a greater aortic annular diameter (Table 1). All 458 patients with SLVSD underwent nonparsimonious propensity matching, resulting in a comparable cohort of AVR and TAVI patients (81 pairs) in terms of the baseline characteristics (Table 2). Nonetheless, among the matched population, the AVR patients still had a slightly larger aortic annular diameter (AVR, $21.6 \pm 2.2 \mathrm{~mm}$ vs TAVI, $22.8 \pm 2.4 ; P=.014$ ), despite a similar valve area and similar mean and peak gradients $(P=.08, P=.10$, and $P=.06$, respectively, Table 2$)$. Furthermore, because of the different "nature" of the compared procedures, AVR were almost always performed with the patient under general anesthesia, with epidural 
TABLE 2. Baseline characteristics of matched population of patients with severe left ventricular systolic dysfunction (LVEF $\leq \mathbf{3 5} \%$ )

\begin{tabular}{|c|c|c|c|}
\hline Variable & $\operatorname{AVR}(\mathbf{n}=\mathbf{8 1})$ & TAVI $(\mathbf{n}=\mathbf{8 1})$ & $P$ value \\
\hline \multicolumn{4}{|c|}{ Demographic and clinical characteristics } \\
\hline Age (y) & $77.6 \pm 6.4$ & $77.5 \pm 10.3$ & .92 \\
\hline Female gender & $31(38.3)$ & $27(33.3)$ & .51 \\
\hline Weight $(\mathrm{kg})$ & $70.2 \pm 16.7$ & $38.7 \pm 14.0$ & .55 \\
\hline Diabetes & $22(27.2)$ & $24(29.6)$ & .12 \\
\hline Creatinine (mg/dL) & $1.5 \pm 1.2$ & $1.5 \pm 1.1$ & .95 \\
\hline Chronic dialysis treatment & $4(4.9)$ & $3(3.7)$ & .70 \\
\hline Albumin $(\mathrm{mg} / \mathrm{dL})$ & $3.4 \pm 1.0$ & $3.6 \pm 0.9$ & .44 \\
\hline Hemoglobin (mg/dL) & $12.1 \pm 1.5$ & $11.7 \pm 1.8$ & .15 \\
\hline Previous AMI & $9(11.1)$ & $3(3.7)$ & .16 \\
\hline Unstable angina & $7(8.9)$ & $3(3.7)$ & .18 \\
\hline COPD & $17(21.0)$ & $23(28.4)$ & .27 \\
\hline Oxygen dependency & $6(7.4)$ & $3(3.7)$ & .30 \\
\hline Neurologic dysfunction & $6(7.4)$ & $5(6.2)$ & .58 \\
\hline Chronic liver disease & $3(3.7)$ & $3(3.7)$ & 1.00 \\
\hline Peripheral arteriopathy & $18(22.2)$ & $21(25.9)$ & .58 \\
\hline Previous cardiac surgery & $19(23.5)$ & $18(22.2)$ & .85 \\
\hline Previous vascular surgery & $3(3.7)$ & $6(7.4)$ & .30 \\
\hline Frailty score & & & .70 \\
\hline 1 (self-sufficient) & $63(77.8)$ & $65(80.3)$ & \\
\hline $\begin{array}{l}2 \text { (partially self- } \\
\text { sufficient) }\end{array}$ & $18(22.2)$ & $16(19.7)$ & \\
\hline Previous PCI & $17(25.3)$ & $29(35.8)$ & .17 \\
\hline $\begin{array}{l}\text { Previous aortic balloon } \\
\text { plasty }\end{array}$ & $9(11.1)$ & $9(11.1)$ & 1.00 \\
\hline Critical preoperative state & $11(13.6)$ & $12(14.8)$ & .82 \\
\hline NHYA class & & & .81 \\
\hline III & $34(42.0)$ & $31(38.3)$ & \\
\hline IV & $15(18.5)$ & $18(22.2)$ & \\
\hline Logistic EuroSCORE & $25.9 \pm 18.8$ & $21.4 \pm 14.3$ & .10 \\
\hline \multicolumn{4}{|c|}{ Angiographic and echocardiographic findings } \\
\hline Coronary artery disease & & & .27 \\
\hline 1 -vessel & $14(17.3)$ & $22(27.2)$ & \\
\hline 2-vessel & $4(4.9)$ & $7(8.6)$ & \\
\hline 3-vessel & $14(17.3)$ & $10(12.3)$ & \\
\hline Left main & - & - & \\
\hline Mitral valve regurgitation & & & .87 \\
\hline None or mild & $49(60.5)$ & $50(61.7)$ & \\
\hline Moderate or severe & $32(39.5)$ & $31(38.3)$ & \\
\hline \multicolumn{4}{|l|}{ Aortic valve pattern } \\
\hline Valve area $\left(\mathrm{cm}^{2}\right)$ & $0.7 \pm 0.3$ & $0.6 \pm 0.2$ & .08 \\
\hline Peak gradient $(\mathrm{mm} \mathrm{Hg})$ & $68.2 \pm 24.7$ & $62.2 \pm 20.9$ & .10 \\
\hline Mean gradient $(\mathrm{mm} \mathrm{Hg})$ & $41.5 \pm 16.4$ & $37.0 \pm 13.1$ & .06 \\
\hline Annulus diameter $(\mathrm{cm})$ & $21.6 \pm 2.16$ & $22.8 \pm 2.45$ & .01 \\
\hline \multicolumn{4}{|l|}{ Procedural characteristics } \\
\hline Urgency status & & & .67 \\
\hline Urgent & $7(8.6)$ & $10(12.3)$ & \\
\hline Emergent & $1(1.2)$ & $1(1.2)$ & \\
\hline Anesthesia & & & $<.01$ \\
\hline General & $80(98.8)$ & $31(38.3)$ & \\
\hline Epidural or local & $1(1.2)$ & $50(61.7)$ & \\
\hline $\begin{array}{l}\text { Associated coronary } \\
\text { procedure }\end{array}$ & $23(28.4)$ & $4(4.9)$ & $<.01$ \\
\hline
\end{tabular}

(Continued)
TABLE 2. Continued

\begin{tabular}{lccc}
\hline Variable & AVR $(\mathbf{n}=\mathbf{8 1})$ & TAVI $(\mathbf{n}=\mathbf{8 1})$ & $\boldsymbol{P}$ value \\
\hline Type of TAVI prosthesis & & & - \\
CV & - & $49(60.5)$ & \\
ELS & - & $32(39.5)$ & \\
\hline
\end{tabular}

Data presented as mean \pm standard deviation or $\mathrm{n}(\%) . L V E F$, Left ventricular ejection fraction; $A V R$, aortic valve replacement; TAVI, transcatheter aortic valve implantation; $A M I$, acute myocardial infarction; $C O P D$, chronic obstructive pulmonary disease; $P C I$, percutaneous coronary intervention; NYHA, New York Health Association; $C V$, CoreValve; $E L S$, Edwards SAPIEN valve.

and/or local anesthetic techniques prevailing with TAVI $(P=.0001$; Table 2$)$. Finally, the AVR patients more frequently underwent concomitant myocardial revascularization $(28.4 \%$ vs $4.9 \%$ for TAVI; $P=.0001$; Table 2$)$.

\section{Outcomes in Matched Pairs}

When the matched patients were analyzed, neither AVR nor TAVI proved superior in terms of 30-day mortality, AMI, stroke, cardiac tamponade, LCOS, and perioperative peak serum creatinine ( $P=$ NS; Table 3$)$. Similarly, the ICU, ImCU, and hospitalization length of stay were comparable between the 2 groups (Table 3 ).

AVR resulted in a significantly lower need for PPM implantation $(1.2 \%$ vs $13.6 \%$ for TAVI; $P=.01)$. In contrast, TAVI demonstrated a significantly lower need for transfusions $(24.4 \%$ vs $76.5 \%$ for AVR; $P<.01)$, despite a comparable mean number of transfusions/patient (AVR, $4.4 \pm 3.8$ vs TAVI, $2.8 \pm 3.7 \mathrm{U} /$ patient; $P=.08$; Table 3), suggesting transfusions were quite uncommon after TAVI but were substantial—and probably "massive"-whenever they occurred.

Finally, when the hemodynamic performance of AVR was compared with that of TAVI, a significantly greater postoperative mean gradient was found in the surgical cohort. In contrast, TAVI showed significantly greater mild-to-severe residual aortic regurgitation $(P<.01$; Table 3).

\section{DISCUSSION}

The main findings of the present study were that TAVI is a comparable alternative to AVR, at least in the short term, for patients with SLVSD, and that both treatments have acknowledged procedure-specific characteristics, with a greater incidence of PPM implantation and residual aortic regurgitation after TAVI and a greater need for transfusions with slightly greater mean gradients after AVR.

Despite the widened application of TAVI technology to an exponential population of patients, few studies have focused on the effect of TAVI in patients with SLVSD. Furthermore, these studies have generally compared the outcomes of patients with low versus normal 
TABLE 3. Hospital outcomes of matched patients

\begin{tabular}{llcc}
\hline \multicolumn{1}{c}{ Variable } & AVR $(\mathbf{n}=\mathbf{8 1})$ & TAVI $(\mathbf{n}=\mathbf{8 1})$ & $\boldsymbol{P}$ value \\
\hline Hospital mortality & $7(8.6)$ & $5(6.2)$ & .37 \\
Perioperative AMI & $0(0)$ & $1(1.2)$ & .55 \\
Stroke & $4(4.9)$ & $1(1.2)$ & .36 \\
LCOS & $9(11.4)$ & $5(6.4)$ & .27 \\
Major vascular complications & $0(0)$ & $1(1.2)$ & .55 \\
Cardiac tamponade & $4(5.1)$ & $1(1.2)$ & .17 \\
Peak postoperative & $1.8 \pm 1.4$ & $1.7 \pm 1.4$ & .57 \\
$\quad$ creatinine (mg/dL) & & & \\
Transfusions/patient (n) & $4.4 \pm 3.8$ & $2.8 \pm 3.7$ & .08 \\
ICU length of stay (d) & $3(2.4-3.7)$ & $2(1.5-2.5)$ & .34 \\
ImCU length of stay (d) & $0(0-0.2)$ & $0(0-0.2)$ & .94 \\
Hospitalization (d) & $14(13.3-14.7)$ & $11(8.8-13.2)$ & .51 \\
Favors AVR & & & \\
$\quad$ PPM implantation (\%) & $1(1.2)$ & $11(13.6)$ & .01 \\
Favors TAVI & & & \\
$\quad$ Transfusion (\%) & $62(76.5)$ & $23(24.4)$ & $<.01$ \\
Echocardiographic findings & & & \\
$\quad$ Mean transprosthetic & $14.5 \pm 8.3$ & $9.6 \pm 5.5$ & $<.01$ \\
$\quad$ gradient (mm Hg) & & & \\
$\quad$ Aortic regurgitation & & & \\
$\quad$ Mild & $13(16.0)$ & $36(44.4)$ & \\
$\quad$ Moderate-to-severe & $7(8.6)$ & $5(6.2)$ & \\
\hline
\end{tabular}

Data presented as n (\%), median ( $95 \%$ confidence interval), or mean \pm standard deviation. AVR, Aortic valve replacement; TAVI, transcatheter aortic valve implantation; $A M I$, acute myocardial infarction; $L C O S$, low cardiac output syndrome; $I C U$, intensive care unit; $\operatorname{ImCU}$, intermediate care unit; $P P M$, permanent pacemaker.

LVEF. ${ }^{4,5,7,13,14}$ Direct comparisons with AVR populations have rarely been reported and have usually been through retrospective analysis of "historical" surgical populations with case-control matching. ${ }^{6}$ A recent study reported the 2-year results of the randomized PARTNER trial, with the advantages of randomization ${ }^{8}$ but lacking the mirror of "real world" practice, which can only come from registry data. Furthermore, some of those studies indirectly derived the preoperative systolic dysfunction from the recognition of low-flow, low-gradient SAS. ${ }^{8,13,14}$ Finally, most of them were single-center ${ }^{13}$ or 2 -center ${ }^{4-7}$ studies.

Thus, the present study represents the first direct comparison of AVR and TAVI in a cohort of patients with SLVSD, whose baseline potential confounding factors were eliminated using nonparsimonious propensity matching. Moreover, the analysis used data from a multicenter contemporary registry enrolling "all-comers" from "realworld" practice. ${ }^{9-11}$

The main finding of the present study was that hospital mortality was not different between AVR and TAVI in patients with SLVSD, confirming the subgroup analysis of patients with a LVEF $<55 \%$ in PARTNER trial A, which preliminary reported comparable 1-year survival between AVR and TAVI. ${ }^{15}$ However, additional investigations of patients with severe ventricular dysfunction were lacking in PARTNER trial A. ${ }^{15}$ The recent report from PARTNER trial A addressing the best treatment option in this high-risk category of patients considered all patients with low-flow aortic stenosis, regardless of the degree of systolic dysfunction. ${ }^{8}$ It also did not differentially analyze patients with low-flow aortic stenosis, those with low-flow aortic stenosis and a low LVEF, and those with low-flow, low-gradient, and low LVEF. ${ }^{8}$ Furthermore, a "high" threshold of LVEF of $50 \%$ was considered the cutoff to define systolic dysfunction in PARTNER trial A. ${ }^{8,15}$ Similarly, a recent 2-center European study reported a comparable 1-year outcome of AVR and TAVI in patients with LVEF $<50 \%$. ${ }^{6}$ The same study demonstrated a comparable procedural success rate and in-hospital and 1-year survival between patients with a normal and depressed LVEF undergoing TAVI, with only lower 1-year freedom from cumulative cardiovascular events in patients with a LVEF $<50 \%$ after TAVI. ${ }^{6}$ Furthermore, some studies analyzing the outcome of TAVI with and without SLVSD demonstrated, not only comparable mortality, but also similar major morbidity. ${ }^{4-7}$ In more detail, Pilgrim and colleagues ${ }^{4}$ showed a similar incidence of myocardial infarction, major stroke, life-threatening bleeding, major access site complications, valvular reintervention, and renal failure at 30 days. van der Boon and colleagues ${ }^{5}$ showed a comparable combined safety endpoint, including myocardial infarction, cerebrovascular events, vascular and bleeding complications, and acute kidney injury. Ewe and colleagues ${ }^{6}$ reported a similar incidence of stroke, heart failure, and cardiovascular events during hospitalization. Fraccaro and colleagues $^{7}$ demonstrated a comparable incidence of AMI, stroke, acute kidney injury, and sepsis. Finally, the German multicenter registry reported a comparable cerebrovascular events rate in patients undergoing TAVI with or without low-flow, low-gradient SAS but also a greater incidence of low output syndrome in the former subgroup. ${ }^{14}$ All these studies also reported the efficacy of TAVI in terms of the risk of major morbidity in the subset of patients with a low LVEF. Thus, with that perspective and given the lack of published data directly comparing the 2 techniques of valve replacement in terms of morbidity, we can interpret our data as underscoring a substantial equipoise between AVR and TAVI in terms of major morbidity (AMI, stroke, cardiac tamponade, LCOS, and perioperative peak serum creatinine). Accordingly, it was not surprising that the ICU, ImCU, and hospitalization stay were also comparable between the 2 procedures in the matched patients with a similar risk profile. The published data have generally favored TAVI versus AVR in terms of the ICU length of stay ${ }^{15,16}$ and hospitalization. ${ }^{15,17}$ However, it has been demonstrated that differences in terms of the baseline characteristics, institutional protocols for ICU, ImCU, and hospital admission and discharge, and periprocedural complications of the individual studies can significantly affect the length of stay after the 2 procedures. ${ }^{10-15,17}$ Hence, a substantial similarity in terms of hospitalization 
has been reported in a recent propensity-matched German experience comparing AVR and TAVI and also resulting in similar hospital outcomes. ${ }^{16}$ We have previously reported similar lengths of hospitalization and ICU stays in 2 analyses of the OBSERVANT registry, with 1 investigating an intermediate-risk propensity-matched population ${ }^{9}$ and 1, a propensity-matched female population undergoing AVR versus TAVI. ${ }^{10}$ Therefore, the present data have confirmed that similar baseline risk profiles and similar hospital mortality and major morbidity will also result in similar ICU, ImCU, and hospital lengths of stay. These data should also be interpreted in light of the supposed superiority of TAVI in terms of cost-effectiveness and length of hospitalization.

AVR resulted in a greater rate of periprocedural transfusions despite a similar transfusion need/patient. The "common" need for transfusions after surgery is well-known and is intrinsically related to the need for cardiopulmonary bypass, with its hemodilution, generation of thrombin, inflammatory reaction, postoperative bleeding, and so forth. ${ }^{18}$ A low preoperative hemoglobin and older age have been reported to predict the need for transfusion after AVR. ${ }^{19}$ Aortic valve stenosis has also been recognized as a hemodynamic condition whose related shear stress favors an acquired von Willebrand syndrome, affecting platelet function and hemostasis. ${ }^{20}$ Moreover, different series comparing AVR and TAVI have previously reported a greater transfusion rate after surgery, ${ }^{9,16}$ especially in female patients. ${ }^{10}$ All these data, together with a relatively low preoperative hemoglobin level and relatively greater prevalence of women in our AVR propensity-matched population, could help to explain that finding. The number of transfusions/patient did not differ between the 2 groups, suggesting that transfusions were quite uncommon after TAVI but were "substantial" at the least when required. The $1.2 \%$ incidence of major vascular complications in our TAVI series (compared with a $0 \%$ incidence with AVR) might explain this finding, owing to the demonstration of a strict relationship between major vascular complications or disabling bleeding and the need for massive transfusions after TAVI. ${ }^{21}$ The same correlation has been previously reported in another OBSERVANT subanalysis. ${ }^{11}$ These data underscore the urgent need for advances in blood management after surgery and for an improved "design" of TAVI prostheses to reduce the risk of major vascular complications.

However, TAVI resulted in a greater need for PPM implantation than did AVR. The need for PPM implantation is a well-known Achilles' heel of TAVI procedures. It has been reported to occur in $15.6 \%$ of the French population enrolled in the French Transcatheter Aortic Valve Intervention (FRANCE-2) study. ${ }^{22}$ Also, in studies investigating a low LVEF population, PPM implantation was required in $18 \%, 26 \%$, and $27 \%$ of patients with SLVSD. ${ }^{4,5,7} \mathrm{We}$ have also previously reported that female gender was an independent predictor of the avoidance of PPM after TAVI, ${ }^{11}$ suggesting that the prevalence of male gender $(66.6 \%)$ in the present cohort of propensity-matched TAVI patients favored the need for PPM implantation. Moreover, different studies have reported the CoreValve prosthesis (Medtronic, Minneapolis, Minn) to be associated with a significantly greater need for PPM implantation owing to its intrinsic design with the stent directly impinging on the membranous septum and the left ventricular outflow tract. ${ }^{17,22-24}$ Again, the $66.7 \%$ prevalence of CoreValve (Medtronic) use in the present TAVI cohort explains our $13.6 \%$ rate of PPM implantation. Finally, we have confirmed the results of the only published report specifically comparing AVR and TAVI in terms of PPM need, which clearly reported a lower periprocedural development of complete atrioventricular block after surgery. ${ }^{17}$

Finally, when the hemodynamic behavior of AVR and TAVI was compared, AVR resulted in an average $5 \mathrm{~mm}$ $\mathrm{Hg}$ greater mean transprosthetic gradient compared with TAVI, confirming our previous experience ${ }^{9,10}$ and that of others. ${ }^{17,24}$ These gradients reflect the different nature between surgical and percutaneous prostheses, with the former preferentially "stented" and the latter exclusively "stentless," without any sewing line and with the need for iatrogenic distension of the aortic annulus by systematic oversizing. ${ }^{24}$ Whether a slightly greater mean gradient in the absence of patient-prosthesis mismatch might affect the outcomes requires long-term outcomes data to determine. Similarly, the greater residual postprocedural aortic regurgitation observed in the TAVI cohort (about $50 \%$ of the patients with SLVSD demonstrated mild-to-severe postprocedural regurgitation) requires long-term data to analyze its effect on the outcomes. Nevertheless, postprocedural regurgitation is another well-known Achilles' heel of TAVI. Significant postprocedural regurgitation was observed in $64.5 \%$ of TAVI patients in the FRANCE- 2 registry. ${ }^{22}$ A recent comprehensive review of the PARTNER trial reported that paravalvular leak after TAVI was associated with increased mortality, even when mild. ${ }^{25}$ Of utmost importance, Fraccaro and colleagues, ${ }^{7}$ specifically analyzing the population of patients with SLVSD undergoing TAVI, reported greater mortality in patients with ventricular dysfunction and found it to be significantly affected by residual moderate-to-severe residual aortic regurgitation. Finally, regarding the need for PPM implantation, the CoreValve prosthesis (Medtronic) has also been reported to induce greater postprocedural aortic regurgitation than Edwards SAPIEN valves (Edwards Lifesciences, Irvine, Calif) ${ }^{26}$ thus suggesting a prosthetic-related worsening of outcome in terms of residual regurgitation in our TAVI cohort. However, despite the need for long-term data, in particular to address the specific effect of mild 
residual regurgitation on outcome, the reported different hemodynamic profile of AVR and TAVI in our patients with SLVSD did not affect the 30-day mortality, 30-day incidence of LCOS, or the ICU, ImCU, or hospital lengths of stay. However, additional studies addressing these topics are warranted.

It was surprising that AVR did not perform better than TAVI in patients with a LVEF of $\leq 35 \%$ when only moderate-to-severe postprocedural aortic regurgitation was considered. That unexpected finding was new in our experience, given that a significant superiority of AVR over TAVI in terms of that endpoint has been previously reported in several OBSERVANT studies comparing a propensity-matched, intermediate-risk population, ${ }^{9}$ a propensity-matched female population, ${ }^{10}$ or genderrelated differences in risk profiles and outcomes. ${ }^{11}$ A recent study comparing AVR and TAVI for patients with a reduced LVEF has confirmed the well-known notion that AVR significantly reduces the aortic regurgitation grade compared with baseline, a finding not confirmed after TAVI. ${ }^{27}$ However, a mean postoperative aortic regurgitation grade of $0.5 \pm 0.3$ after AVR was also reported in that study. ${ }^{27}$ Furthermore, a report from the Veterans Affairs Hospital clearly revealed that postoperative residual regurgitation after standard AVR is more common than expected ( $13 \%$ of aortic regurgitation of any degree in their study), especially when severe calcifications are encountered, after biologic prosthesis placement, in aged patients, or in those with a small body surface area, ${ }^{28}$ all typical features of any contemporary frail and elderly population undergoing AVR, such as the population included in the present study. A recent Canadian surgical experience also confirmed the nonrare occurrence of residual regurgitation after standard AVR and its prognostic significant on survival when severe. ${ }^{29}$ Although an "imbalance" toward the "worst AVR patients" could not be excluded in the present study because of the use of propensity score matching (which "couples" AVR patients to similar TAVI patients), this unexpected finding deserves additional investigation. It also requires investigation because of the anticipated superiority of AVR over TAVI in terms of moderate-to-severe postprocedural aortic regurgitation.

\section{Study Limitations}

The main limitation of the present study was related to the observational nature of the OBSERVANT registry. Evaluating the effect of a specific treatment using a registry can lead to incorrect conclusions because of the influence of unknown confounding variables. However, treatment was not assigned randomly but after evaluation by a multidisciplinary local "heart team," as suggested by current guidelines. ${ }^{12}$ This policy could still have generated an unavoidable risk of bias regarding treatment selection. Also, no standardized protocols for cardiopulmonary bypass or anesthetic techniques were used because of the observational nature of the registry. ${ }^{9-11}$ Also, details on preoperative stratification, treatment allocation, techniques used (eg, surgery or TAVI, transfemoral vs nontransfemoral routes, choice of surgical and percutaneous valves), definition of outcome variables, postprocedural medical management, data management, and quality control of the registry have all been previously reported. ${ }^{9-11}$ In contrast, registries are considered the "real-world" mirror of current practice, and their results have witnessed the contemporary outcomes after TAVI and AVR in so-called all-comer patients. Moreover, the nonparsimonious propensitymatched analysis used in the present study has significantly contributed to attenuate the potential bias. Finally, the absence in published data of direct comparisons between AVR and TAVI in "all-comer" patients with SLVSD has emphasized the significance of these preliminary results.

The endpoints were not perfectly stringent with the VARC/VARC-2 criteria. $^{12}$ In particular, we did not detail the causes of mortality according to the VARC-2 criteria, ${ }^{12}$ because the individual causes of death could not be precisely identified from the registry. However, 30-day mortality has been universally recognized as mortality directly related to the index procedure; thus, it could be considered similar to the recently defined "procedural mortality" of the VARC-2 criteria. ${ }^{12}$ When the OBSERVANT registry was launched, neither the VARC nor VARC-2 criteria were available. ${ }^{12}$ Furthermore, the VARC definitions were specifically designed to define the complications after TAVI; therefore, an overestimation of adverse events in the AVR population could have occurred. ${ }^{9-12}$

\section{CONCLUSIONS}

Our study has demonstrated that both AVR and TAVI can achieve good results in patients with SLVSD, without any technique-related early substantial survival benefit. Also, in this subgroup of patients, the original weaknesses of both procedures have been confirmed. These weaknesses include the cardiopulmonary bypass-related hemodilution and inflammation cascade-with its consequent need for transfusions-for the surgical cohort and the excessive need for PPM implantation and the still high prevalence of residual regurgitation, the effect of which on severely depressed ventricles could become detrimental over time for the TAVI cohort. From our experience and the comparable results, the presence of SLVSD should not represent, on its own, an argument to shift a patient with SAS considered for surgery to TAVI.

\section{References}

1. Barili F, Pacini D, Capo A, Ardemagni E, Pellicciari G, Zanobini M, et al. Reliability of new scores in predicting perioperative mortality after isolated aortic valve surgery: a comparison with the Society of Thoracic Surgeons score and logistic EuroSCORE. Ann Thorac Surg. 2013;95:1539-44. 
2. Tribouilloy C, Levy F, Rusinaru D, Guéret P, Petit-Eisenmann H, Baleynaud S, et al. Outcome after aortic valve replacement for low-flow/low-gradient aortic stenosis without contractile reserve on dobutamine stress echocardiography. J Am Coll Cardiol. 2009;53:1865-73.

3. Iung B, Cachier A, Baron G, Messika-Zeitoun D, Delahave F, Tornos P, et al. Decision-making in elderly patients with severe aortic stenosis: why are so many denied surgery? Eur Heart J. 2005;26:2714-20.

4. Pilgrim T, Wenasewer P, Meuli F, Huber C, Stortecky S, Seiler C, et al. Clinical outcome of high-risk patients with severe aortic stenosis and reduced left ventricular ejection fraction undergoing medical treatment or TAVI. PLoS One. 2011;6:e27556.

5. van der Boon RM, Nuis RJ, Van Mieghem NM, Benitez LM, van Geuns RJ, Galema TW, et al. Clinical outcome following transcatheter aortic valve implantation in patients with impaired left ventricular systolic function. Catheter Cardiovasc Interv. 2012;79:702-10.

6. Ewe SH, Marsan NA, Pepi M, Delgado V, Tamborini G, Muratori M, et al. Impact of left ventricular systolic dysfunction on clinical and echocardiographic outcomes following transcathter aortic valve implantation for severe aortic stenosis. Am Heart J. 2010;160:1113-20.

7. Fraccaro C, Al-Lamee R, Tarantini G, Maisano F, Napodano M, Montorfano M, et al. Transcatheter aortic valve implantation in patients with severe left ventricular dysfunction: immediate and mid-term results, a multicenter study. Circ Cardiovasc Interv. 2012;5:253-60.

8. Herrmann HC, Pibarot P, Hueter I, Gertz ZM, Stewart WJ, Kapadia S, et al. Predictors of mortality and outcomes of therapy in low-flow severe aortic stenosis: a Placement of Aortic Transcatheter Valves (PARTNER) trial analysis. Circulation. 2013;127:2316-26.

9. D'Errigo P, Barbanti M, Ranucci M, Onorati F, Covello RD, Rosato S, et al. Transcatheter aortic valve implantation versus surgical aortic valve replacement for severe aortic stenosis: results from an intermediate risk propensity-matched population of the Italian OBSERVANT study. Int J Cardiol. 2013;167:1945-52.

10. Onorati F, D'Errigo P, Barbanti M, Rosato S, Covello RD, Maraschini A, et al. Results differ between trans-aortic and open surgical aortic valve replacement in women: a propensity-matched study of the Italian OBSERVANT registry. Ann Thorac Surg. 2013;96:1336-42.

11. Onorati F, D'Errigo P, Barbanti M, Rosato S, Covello RD, Maraschini A, et al. OBSERVANT Investigators. Different impact of female gender on baseline characteristics and major periprocedural outcomes of transcathter and surgical aortic valve interventions: results of the multicenter Italian OBSERVANT registry. J Thorac Cardiovasc Surg. July 13, 2013 [Epub ahead of print].

12. Kappetein AP, Head SJ, Genereux P, Piazza N, van Mieghem NM, Blackstone EH, et al. Updated standardized endpoint definitions for transcatheter aortic valve implantation: the Valve Academic Research Consortium-2 consensus document. J Thorac Cardiovasc Surg. 2013;145:6-23.

13. Gotzmann M, Lindstaedt M, Bojara W, Ewers A, Mugge A. Clinical outcome of transcatheter aortic valve implantation in patients with low-flow low-gradient aortic stenosis. Catheter Cardiovasc Interv. 2012;79:693-701.

14. Lauten A, Zhan R, Horack M, Sievert H, Linke A, Ferrari M, et al. Transcatheter aortic valve implantation in patients with low-flow, low-gradient aortic stenosis. J Am Coll Cardiol Intv. 2012;5:552-9.

15. Stohr R, Dohmen G, Herpetz R, Brehmer K, Aktug O, Koos R, et al. Thirty-day outcome after transcatheter aortic valve implantation compared with surgical valve replacement in patients with high-risk aortic stenosis: a matched comparison. Coron Artery Dis. 2011;22:595-600.
16. Conradi L, Seiffert M, Treede H, Silaschi M, Baldus S, Schirmer J, et al. Transcatheter aortic valve implantation versus surgical aortic valve replacement: a propensity score analysis in patients at high surgical risk. J Thorac Cardiovasc Surg. 2012;143:64-71.

17. Bagur R, Rodes-Cabau J, Gurvitch R, Dumont E, Velianou JL, Manazoni J, et al Need for permanent pacemaker as a complication of transcatheter aortic valve implantation and surgical aortic valve replacement in elderly patients with severe aortic stenosis and similar baseline electrocardiographic findings. J Am Coll Cardiol Intv. 2012;5:540-51.

18. Boisclair MD, Lane DA, Philippou H, Esnouf MP, Sheikh S, Hunt B, et al. Mechanisms of thrombin generation during surgery and cardiopulmonary bypass. Blood. 1993;82:3350-7.

19. Dial S, Delabays E, Albert M, Gonzalez A, Camarda J, Law A, et al. Hemodilution and surgical hemostasis contribute significantly to transfusion requirements in patients undergoing coronary artery bypass. J Thorac Cardiovasc Surg. 2005; 130:654-61.

20. Panzer S, Badr Eslam R, Schneller A, Kaider A, Koren D, Eichelberger B, et al Loss of high-molecular-weight von Willebrand factor multimers mainly affects platelet aggregation in patients with aortic stenosis. Thromb Haemost. 2010; 103:408-14.

21. Tchetche D, van der Boon RMA, Dumonteil N, Chieffo A, Van Mieghem NM, Farah B, et al. Adverse impact of bleeding and transfusion on the outcome post-transcatheter aortic valve implantation: insights from the PooledRotterdAm-Milano-Toulouse In Collaboration Plus (Pragmatic Plus) initiative. Am Heart J. 2012;164:402-9.

22. Gilard M, Eltchaninoff H, Iung B, Donzeau-Gouge P, Chevreul K, Fajadet J, et al., FRANCE-2 Investigators. Registry of transcatheter aortic-valve implantation in high risk patients. $N$ Engl J Med. 2012;366:1705-15.

23. Ledwoch J, Franke J, Gerckens U, Kuck KH, Linke A, Nickenig G, et al. Incidence and predictors of permanent pacemaker implantation following transcatheter aortic valve implantation: analysis from the German transcatheter aortic valve interventions registry. Catheter Cardiovasc Interv. 2013;82:E569-77.

24. Clavel MA, Webb JG, Pibarot P, Altwegg L, Dumont E, Thompson C, et al. Comparison of the hemodynamic performance of percutaneous and surgical bioprostheses for the treatment of severe aortic stenosis. J Am Coll Cardiol. 2009; 53:1883-91.

25. Svensson LG, Tuzcu M, Kapadia S, Blackstone EH, Roselli EE, Gillinov MA, et al. A comprehensive review of the PARTNER trial. J Thorac Cardiovasc Surg. 2013;145(Suppl):S11-6.

26. Watanabe Y, Hayashida K, Yamamoto M, Mouillet G, Chevalier B, Oguri A, et al Transfemoral aortic valve implantation in patients with an aortic annulus dimension suitable for either the Edwards valve or the CoreValve. Am J Cardiol. 2013; 112:707-13.

27. Bauer F, Coutant V, Bernard M, Stepowski D, Tron C, Cribier A, et al. Patients with severe aortic stenosis and reduced ejection fraction: earlier recovery of left ventricular systolic function after transcatheter aortic valve implantation compared with surgical valve replacement. Echocardiography. 2013;30:865-70.

28. O'Rourke DJ, Palac RT, Malenka DJ, Marrin CA, Arbuckle BE, Plehn JF. Outcome of mild periprosthetic regurgitation detected by intraoperative transesophageal echocardiography. J Am Coll Cardiol. 2001;38:163-6.

29. Sponga S, Perron J, Dagenais F, Mohammadi S, Baillot R, Doyle D, et al. Impact of residual regurgitation after aortic valve replacement. Eur J Cardiothorac Surg. 2012;42:486-92 